\title{
REFLEXIONES SOBRE LA INCLUSIÓN FINANCIERA EN EL ÁMBITO INTERNACIONAL: EVOLUCIÓN Y NUEVOS DESAFÍOS
}

\author{
Autora: Susana de los Ríos Sastre ${ }^{1}$ \\ Profesora Propia Adjunta del Departamento de Gestión Financiera \\ Facultad de Ciencias Económicas y Empresariales \\ Universidad Pontificia Comillas
}

\section{Resumen}

La inclusión financiera ocupa un lugar primordial en las agendas de numerosos organismos internacionales y de los gobiernos de muchos países del mundo, principalmente debido a su potencial influencia positiva sobre la reducción de la pobreza, el crecimiento económico y la estabilidad del sistema financiero. En los últimos años, gracias a un esfuerzo conjunto, se ha reducido el grado de exclusión financiera de algunos segmentos de la población mundial. No obstante, aún queda mucho trabajo por hacer. Este artículo presenta una revisión sintética de aspectos relevantes en materia de inclusión financiera, que incluye: las diferentes dimensiones del concepto, los posibles factores determinantes y la evolución reciente de una serie de in-

\footnotetext{
${ }^{1}$ srios@icade.comillas.edu
} 
dicadores de inclusión financiera. Asimismo, se exponen cuáles son los principales desafíos a los que se enfrentan las autoridades públicas en el ámbito internacional.

Palabras clave: inclusión financiera; no bancarizado; exclusión financiera; educación financiera.

\title{
Financial Inclusion around the World: progress and challenges
}

\begin{abstract}
Financial inclusion is becoming a priority for many national governments and international institutions across the world, mainly because of its potential positive influence on the reduction of poverty, and the improvement of economic growth and financial stability. Fortunately, the efforts to increase the level of financial inclusion have started to bear fruit. However, there is still work to be done. This article presents an overview of important issues of financial inclusion, which includes the different dimensions of the concept and its determinants, and recent advances in this field. It also presents some reflections on the main challenges facing public authorities and financial policymakers.
\end{abstract}

Key words: financial inclusion; unbanked; financial exclusion; financial literacy.

Recibido: 19/07/2016

Aceptado: 25/07/2016

\section{INTRODUCCIÓN}

La inclusión financiera se ha convertido en uno de los puntos clave en la mayoría de las agendas de las instituciones públicas, al reconocer de forma generalizada su importancia para lograr una estabilidad financiera e impulsar un crecimiento económico inclusivo y sostenible en el ámbito internacional ${ }^{2}$. No obstante, según los últimos datos publicados por el Banco Mundial³, casi dos mil

\footnotetext{
${ }^{2}$ Un resumen de los 17 objetivos establecidos en la "Agenda 2030" para un desarrollo sostenible, en el marco de Naciones Unidas, está disponible en http://www.un.org/sustainabledevelopment/sustainabledevelopment-goals.

${ }^{3}$ Consúltese a este respecto la página web del Banco Mundial, que ofrece información completa y actualizada de las diferentes estrategias internacionales en materia de inclusión financiera, así como datos nacionales y globales sobre los avances de los diferentes planes de acción en este campo (http://www. worldbank.org/en/topic/financialinclusion).
} 
millones de personas en el mundo no tienen acceso a servicios financieros formales y se estima que alrededor de un $73 \%$ de los excluidos financieramente hablando están concentrados en 25 países. En la figura 1, se muestra una panorámica mundial del porcentaje de personas adultas (con edad superior a los 15 años) que tiene cuenta en una entidad financiera. Por tener alguna cifra de referencia, la media mundial se sitúa alrededor del $62 \%$ y como puede observarse en dicha figura hay una gran cantidad de países (son los sombreados en color oscuro) que están por debajo de ese promedio, principalmente aquellos con menor nivel de riqueza.

\section{Figura 1. Población adulta con cuenta en una entidad financiera (año 2014)}

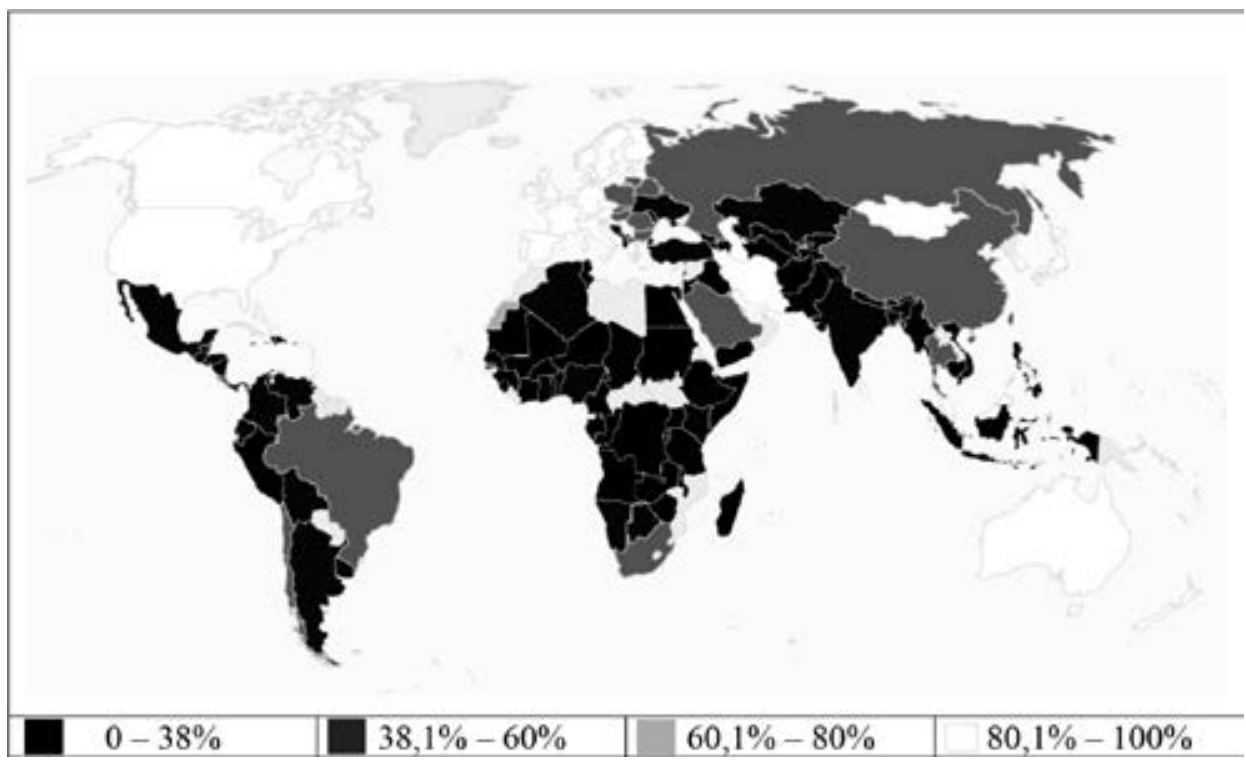

Fuente: Global Findex data 2014, disponibles en http://datatopics.worldbank.org/financialinclusion

Recordemos que hace unos años el Grupo del Banco Mundial, apoyado por sus socios, estableció un ambicioso objetivo para conseguir un acceso financiero universal para todos los adultos en edad de trabajar con un horizonte temporal fijado en 2020 (Universal Financial Access - UFA 2020). La iniciativa UFA $2020^{4}$ impli-

${ }^{4}$ Los trabajos relacionados con el objetivo UFA 2020 se concentran en los 25 países con un porcentaje muy alto de la población en exclusión financiera. India y China son los países con una proporción mayor de "no bancarizados" a nivel mundial (representan un 32\% del total), pero la prioridad de actuación también se dirige a otros países como son: Brasil, Colombia, Etiopía, Indonesia, Kenia, Méjico, Marruecos, Nigeria, Pakistán, Perú, Filipinas, Tanzania, Turquía o Zambia, entre otros. 
ca en la práctica que cualquier adulto posea una cuenta en una entidad financiera (o un instrumento electrónico) para ahorrar, realizar pagos o recibir cobros. Por supuesto, se considera que este acceso universal a los servicios financieros ayudaría a cumplir el objetivo global de poner fin a la pobreza extrema antes de 2030, al tiempo que ayudaría a aumentar la prosperidad en el mundo.

No obstante, a pesar de que la evidencia muestra los beneficios de tener acceso a los servicios financieros básicos (ahorro, pagos y seguro) para cualquier persona, desde el Banco Mundial se advierte que la inclusión financiera no debería traducirse en financiación para todos y a toda costa. Por ejemplo, no tiene sentido crear millones de nuevas cuentas en entidades financieras, si no se van a usar de forma regular (Demirgüç-Kunt et al., 2015). Más bien se trata de promover una inclusión financiera responsable, lo cual requiere que se produzcan cambios por las dos vertientes, es decir, por el lado de la oferta y de la demanda. El objetivo debería ser facilitar el acceso a productos/servicios financieros que se adapten a las necesidades de los clientes, con fijación de precios razonables y asequibles que permitan reducir los fallos del mercado, al tiempo que los consumidores mejoran sus conocimientos y habilidades financieras para poder tomar decisiones con mayor criterio. Se trata, por tanto, hay que entender la inclusión financiera como un concepto multidimensional.

Es cierto que hay numerosas instituciones públicas y privadas, nacionales e internacionales, que están trabajando con la finalidad de reducir los niveles de exclusión financiera mundial y, en principio, cualquier contribución puede ser positiva. Sin embargo, sin ánimo de menospreciar ninguna de las iniciativas o acciones en esta materia, el papel activo del sector público es esencial. De hecho, se enfrentan a importantes desafíos que van desde el desarrollo de medidas adecuadas de protección al consumidor, hasta el establecimiento de programas efectivos de educación financiera, sin olvidar el aprovechamiento de las innovaciones tecnológicas.

\section{CONCEPTO DE INCLUSIÓN FINANCIERA Y SU MEDICIÓN}

A pesar de ocupar un lugar primordial en las políticas públicas de un gran número de países y de su consideración como factor clave para conseguir un sistema financiero estable y eficiente, hay en la literatura diferentes definiciones para inclusión financiera. Veamos algunas de las propuestas desde distintos organismos internacionales.

Por ejemplo, la Organización para la Cooperación y el Desarrollo Económicos (OCDE) a través de la International Network on Financial Education (INFE) propone una primera aproximación al término, definiendo inclusión financiera como: "el proceso de promoción de un acceso asequible, oportuno y adecuado a una amplia gama de productos y servicios financieros regulados y la ampliación de su uso hacia todos los segmentos de la sociedad”. Además, añaden a la defini- 
ción como objetivo final, la contribución al bienestar financiero y a la inclusión económica y social (Atkinson y Messy, 2013).

Por su parte, la Global Partnership for Financial Inclusion (GPFI) 5 se refiere a inclusión financiera como la situación en la cual los adultos en edad de trabajar tienen acceso efectivo a los servicios financieros (crédito, ahorro, pagos y seguros) proporcionados por instituciones financieras formales. Entendiendo por acceso efectivo una prestación del servicio apropiada y responsable, lo cual implica que los costes son asumibles para los usuarios y sostenibles para las instituciones que los ofrecen, lo que permitiría que la población financieramente excluida utilice los servicios financieros formales (GPFI, 2011). Estas ideas están recogidas en cierta medida en los principios para la inclusión financiera innovadora propuestos por los líderes del G-20 en 2010, y que pueden resumirse en los siguientes puntos (G-20, 2010):

- Liderazgo: fomentar un compromiso del gobierno hacia la inclusión financiera para ayudar a aliviar la pobreza.

- Diversidad: poner en práctica los enfoques de políticas que promuevan la competencia, y ofrecer incentivos de mercado para la distribución de acceso sostenible a las finanzas y el uso de una amplia gama de servicios a precios asequibles (ahorro, crédito, pagos, transferencias, seguros), así como una diversidad de proveedores de servicios.

- Innovación: promover la innovación tecnológica e institucional como un medio para ampliar el acceso y la utilización del sistema financiero, incluso haciendo frente a las deficiencias de infraestructura.

- Protección: fomentar un enfoque global de la protección al consumidor, que reconozca el papel del gobierno, los proveedores y los consumidores.

- Empoderamiento: desarrollar la alfabetización financiera y la capacitación financiera.

- Cooperación: crear un entorno institucional con líneas claras de rendición de cuentas y la coordinación dentro del gobierno, y fomentar la cooperación y de consultas directas a través del gobierno, empresas y otros actores.

- Conocimiento: utilizar mejor los datos para medir el progreso.

- Proporcionalidad: generar un marco normativo y reglamentario que sea proporcional a los riesgos y beneficios implicados en este tipo de productos y servicios innovadores.

\footnotetext{
${ }^{5}$ Recordemos que se trata de la plataforma, puesta en marcha por el Grupo de los Veinte (G-20) en diciembre de 2010 con la finalidad de colaborar en materia de inclusión financiera. Denominada en español como "Alianza Mundial para la Inclusión Financiera", pretende unir las acciones de todos los países miembros del G-20, países no miembros y otros stakeholders interesados en llevar a cabo acciones relativas a la inclusión financiera. Para más detalle sobre su actividad, puede consultarse la página web del GPFI: http://www.gpfi.org.
} 
Una versión más abreviada del concepto de inclusión financiera hace alusión al porcentaje de la población con acceso a los servicios financieros formales. En todo caso, sea cual sea la definición elegida para inclusión financiera, en todas ellas hay una coincidencia en las dos dimensiones básicas de la misma, que son: "acceso" y "uso".

Acceso: capacidad de la población en general para acceder a los productos y servicios ofrecidos por el sistema financiero formal. El mayor o menor acceso está influido, entre otras cosas, por las potenciales barreras para abrir una cuenta en una entidad financiera (v.g. costes) o la amplitud de la red de sucursales de las entidades financieras de cada país (u otros puntos de atención al público), así como la distancia geográfica a las mismas.

Uso: se refiere a la regularidad y frecuencia en la utilización de los productos y servicios financieros. Hay que tener en cuenta que el uso, más o menos, intensivo que cada individuo hace de tales servicios, puede verse condicionado por el comportamiento financiero de otros miembros de la unidad familiar.

Ahora bien, encontramos en la literatura otras dimensiones de la inclusión financiera. En otros, la Alliance for Financial Inclusion ${ }^{6}$ (AFI, 2010) contempla dos componentes adicionales: calidad y bienestar.

Calidad: hace alusión a la diversidad de los productos y servicios financieros ofrecidos y a la adaptabilidad de dichos productos y servicios a las necesidades de los clientes. También podría incluirse en este componente el tipo de regulación y supervisión de los servicios financieros disponibles o, incluso, la existencia de políticas de protección al consumidor financiero.

Bienestar: esta dimensión amplía la visión sobre la inclusión financiera y se refiere al impacto que los productos y servicios financieros y su utilización tienen en la vida de los consumidores, es decir, en qué medida provocan cambios en sus hábitos de consumo, en la actividad de sus negocios o en su estado de bienestar.

Por tanto, no hay duda de que la inclusión financiera tiene un carácter multidimensional y está influida por una gran variedad de factores, que pertenecen tanto a la oferta de productos y servicios financieros como a las características de la demanda de los mismos. Tradicionalmente, los factores del lado de la oferta se han relacionado con la dimensión "acceso", mientras que los elementos relativos a la demanda se han identificado con la dimensión "uso". Si bien, no es sencillo realizar una correspondencia tan ajustada. Lo que sí es cierto es que, hasta la fecha y probablemente por la dificultad en la recogida de datos, hay más estudios

${ }^{6}$ La Alliance for Financial Inclusion fue creada en 2008 por la Bill $\mathcal{E}$ Melinda Gates Foundation como una red global para compartir conocimiento en materia de inclusión financiera, por parte de las autoridades públicas y otros reguladores de los sistemas financieros de un conjunto amplio de países en desarrollo. Desde sus comienzos ha tenido un papel muy activo en este ámbito y en la actualidad, reúne a 125 miembros (bancos centrales y otros organismos financieras públicas) de 96 países en desarrollo y emergentes. Una gran variedad de informes, noticias relevantes y otras actividades de AFI están disponibles en http://www.afi-global.org. 
realizados sobre las variables que afectan a la inclusión financiera por el lado de la oferta que por el lado de la demanda?.

Adicionalmente, cada vez con más frecuencia se utiliza el término "bancarización" como sustituto de "inclusión financiera”, pudiendo llevar a confusión si no se aclara apropiadamente. Bancarización no debería entenderse únicamente como la incorporación al sistema bancario formal de segmentos de la población habitualmente desatendidos por la banca tradicional, sino más bien como el acceso y uso de los servicios financieros ofrecidos por las instituciones financieras en general, no sólo por los bancos. En esta línea, hay autores que unen ambos términos al definir inclusión financiera como "el proceso por el cual se maximiza el acceso y el uso de los servicios financieros formales...al tiempo que se mitigan las barreras potenciales, para que individuos cuyo beneficio marginal de estar bancarizados exceda a su coste marginal, puedan acceder a este tipo de servicios sin verse afectados por fallos de mercado" (Cámara y Tuesta, 2015).

\section{FACTORES QUE INFLUYEN EN LA INCLUSIÓN FINANCIERA: EL PAPEL DE LA EDUCACIÓN FINANCIERA}

Como se ha expuesto anteriormente, la inclusión financiera es un concepto multidimensional que se ve afectado por una diversidad de factores, de mayor o menor calado, pertenecientes tanto a la oferta como a la demanda de los servicios financieros. Estos factores se han convertido en verdaderas barreras para que algunos segmentos de la población puedan acceder a los servicios financieros formales. Por el lado de la oferta, la inclusión financiera se ve afectada entre otras razones por: las restricciones regulatorias, las características del propio mercado financiero, la oferta de servicios financieros alternativos o por elementos físicos o geográficos. Por el lado de la demanda, constituyen barreras para la inclusión financiera: la educación, las circunstancias personales (vulnerabilidad), el grado de inclusión social y tecnológica o el nivel de educación económico-financiera.

En la siguiente tabla se resumen, a modo de ejemplo, algunos de los obstáculos con los que se encuentran muchas personas que quieren integrarse en el sistema financiero formal, y sobre los que se está trabajando para reducir el nivel de exclusión financiera de la población mundial.

\footnotetext{
${ }^{7}$ La preocupación por la carencia de una medición completa y comparable de la inclusión financiera en el ámbito internacional y de la correspondiente monitorización de los avances conseguidos, se pone de manifiesto en los trabajos de la OCDE. Es previsible que para finales de 2016 estén disponibles datos actualizados sobre inclusión financiera y educación financiero, siguiendo los criterios recogidos en OECD/INFE (2015). Sobre la medición de los factores determinantes de la inclusión financiera, véanse también: Atkinson y Messy (2013), Demirgüç-Kunt y Klapper (2013) y AFI (2010); así como otros trabajos allí citados.
} 
Tabla 1. Barreras a la inclusión financiera (oferta)

\begin{tabular}{|c|}
\hline $\begin{array}{c}\text { RESTRICCIONES REGULATORIAS } \\
\text { Prohibición o restricciones para que ciertos grupos puedan acceder a los productos/servicios } \\
\text { financieros (jóvenes, inmigrantes) }\end{array}$ \\
\hline OFERTA DE SERVICIOS FINANCIEROS ALTERNATIVOS \\
Servicios de cambio de cheques \\
Prestamistas a domicilio
\end{tabular}

Fuente: elaboración propia basada en Atkinson y Messy (2013).

En paralelo, hay una serie de variables que influyen en el acceso a los servicios financieros desde el lado de la demanda. Las conclusiones recogidas recientemente en el Global Financial Development Report del Banco Mundial, correspondientes a la encuesta realizada por el Financial Development Barometer, muestran que un $78 \%$ de los entrevistados considera que la falta de conocimientos financieros entre los pobres es la mayor barrera para que este grupo de la sociedad pueda acceder a los productos y servicios financieros disponibles en cada país. Al mismo tiempo, un 32\% de los encuestados considera que la educación financiera es la mejor política para mejorar el acceso a la financiación de las personas con bajo nivel de renta, mientras que un $18 \%$ cree que la prioridad está en la mejora del marco legal y un 17\% destaca la promoción de nuevas tecnologías aplicadas al crédito (World Bank, 2014). En esta misma línea, Ghatak (2013) en su intento por cuantificar la influencia de la educación financiera en la inclusión financiera verifica empíricamente que los factores, por el lado de la demanda, con mayor influencia sobre la inclusión financiera son, por este orden: la accesibilidad, la cultura, la educación financiera, el patrimonio y el nivel de renta.

En la siguiente tabla se recogen, de forma esquemática, las principales barreras por el lado de la demanda que, con mayor o menor intensidad, están presentes en todos los países. 
Tabla 2. Barreras a la inclusión financiera (demanda)

\begin{tabular}{|c|}
\hline EDUCACIÓN \\
Bajos niveles en la educación general \\
Niveles limitados de alfabetización/aritmética \\
\hline CIRCUNSTANCIAS PERSONALES \\
Desempleo \\
Falta de recursos/dinero \\
Carencia de historial crediticio \\
\hline EXCLUSION SOCIAL Y TECNOLÓGICA \\
Estatus inmigratorio \\
\hline Falta de familiaridad con el uso de internet, la banca móvil o los cajeros automáticos \\
EDUCACIÓN FINANCIERA \\
Bajos conocimientos y habilidades financieras \\
Carencia de conocimiento de los productos financieros \\
\hline FACTORES CULTURALES/PSICOLÓGICOS \\
Falta de confianza en los servicios financieros \\
Preferencias personales \\
Creencias religiosas
\end{tabular}

Fuente: elaboración propia basada en Atkinson y Messy (2013).

La falta de una educación financiera adecuada se considera uno de los factores determinantes de la exclusión financiera de gran parte de la población mundial. En este sentido, la OCDE ha verificado la existencia de una relación positiva entre la educación financiera y la inclusión financiera, a través de los estudios piloto llevados a cabo en los últimos años. Según se expone en el informe sobre educación e inclusión financiera OECD/INFE (2013), la inclusión financiera se corresponde positivamente con un alto nivel de conocimientos financieros, como puede apreciarse, entre otros, en el indicador que mide el grado de conocimiento que la población tiene de los productos financieros existentes en su país. Asimismo, se verifica la relación directa con ciertos factores sociodemográficos, como son: la edad, el género, el nivel de educación general y el nivel de renta. Estos datos sugieren que los programas de educación financiera dirigidos a ese público objetivo serían bien recibidos (Lusardi y Mitchell, 2013). En este sentido, un número significativo de países en desarrollo o emergentes convencidos de la estrecha relación entre ambas, están buscando una mejora de la inclusión financiera poniendo en marcha Estrategias Nacionales de Educación Financiera (OECD/INFE, 2014).

Desde otros ámbitos, como por ejemplo el Comité Europeo Económico y Social (European Economic and Social Committee), se ha reconocido también que un mayor grado de conocimientos financieros permitiría reducir la exclusión financiera, aunque para que este efecto positivo tenga lugar no debería tratarse únicamente de la transmisión de conocimientos y habilidades financieras, sino 
también lograr que los consumidores sean capaces de tomar decisiones informadas y bien fundamentadas sobre su economía personal (EESC, 2013).

Partiendo de la interconexión entre educación financiera e inclusión financiera, sería interesante identificar mejor el papel de la educación financiera para poder establecer programas más efectivos. El Banco Mundial y la OCDE, entre otros, apuntan que la educación financiera que se imparte en cursos dirigidos a la población general no suele producir un gran impacto y que son las autoridades públicas las que deberían afrontar algunos retos en el diseño de los programas de educación financiera para conseguir que sean realmente eficientes en el logro de la inclusión financiera (World Bank, 2014; Atkinson y Messy, 2013). Entre los retos pendientes en materia de educación financiera están:

- Adaptar los canales de distribución a las características del público objetivo, incluso adecuar el lenguaje utilizado en cada caso. Por una parte, el nivel de educación general de estos grupos de la población suele ser bajo y, por otra, suele ser complicado captar la atención de ese público objetivo, para lo cual está dando buen resultado el uso de programas de radio o televisión.

- Identificar los intermediarios más adecuados para llegar a la población objetivo, gestionando el posible conflicto de intereses, más aún si se colabora con el sector privado. Por ejemplo, cuando es una entidad financiera el intermediario que ofrece programas de educación financiera, los intereses educativos podrían chocar con los comerciales.

- Diferenciar las decisiones financieras individuales de las que se toman en el ámbito familiar, para poder ajustar los programas al público objetivo. La mayoría de las iniciativas de educación financiera tienen van dirigidas a la mejora de los conocimientos y habilidades financieras para que de los individuos tomen decisiones financieras más correctas, sin embargo, cuando el objetivo final es la inclusión financiera la unidad familiar toma más protagonismo.

Ahora bien, tal y como se ha comentado con anterioridad, las políticas más exitosas de inclusión financiera son aquellas que combinan educación financiera, mejor acceso a los mercados y una regulación sólida que proteja al consumidor de servicios financieros. Expresado de otra forma, la protección de los intereses de los consumidores financieros es una parte también fundamental de la inclusión financiera y, por tanto, debería ocupar un lugar primordial en las políticas públicas de cualquier país. Desde la Alliance for Financial Inclusion se reconoce que las intervenciones públicas ${ }^{8}$ en este campo ayudan a incrementar la confianza del

${ }^{8}$ El Grupo de Trabajo de Empoderamiento del Consumidor y Conducta del Mercado (CEMC) de AFI coopera con el Subgrupo de Educación Financiera y Protección al Consumidor del G-20 para desarrollar indicadores de protección al consumidor y las dimensiones de calidad de la inclusión financiera. 
consumidor en el sistema financiero formal, al tiempo que promueven el consumo de servicios financieros, y esta idea se pone de manifiesto en los compromisos adquiridos por sus miembros (AFI, 2013).

Es más, crear nuevas oportunidades en el mercado financiero podría llegar a ser perjudicial si no viene acompañado de un nivel suficiente de educación financiera y se dispone de un mercado financiero regulado de forma adecuada, tal y como lo verifican las experiencias de algunos países entre los que se encuentran: Brasil, India, Indonesia y Méjico (World Bank, 2014).

\section{EVOLUCIÓN DE LA INCLUSIÓN FINANCIERA EN EL ÁMBITO INTERNACIONAL}

Parece ser que los esfuerzos realizados desde numerosos organismos públicos, nacionales e internacionales, han empezado a dar sus frutos, con lo cual se puede hablar de una tendencia positiva en la inclusión financiera en términos mundiales; aunque se mantienen las disparidades entre países y hay una gran diversidad en las características individuales de los usuarios de servicios financieros (AFI, 2013; OECD/INFE, 2013; Demirgüç-Kunt et al., 2015).

En el año 2011 se estimaba que había unos 2.500 millones de personas sin acceso a los servicios financieros, que pertenecían mayoritariamente a los segmentos de la población menos favorecidos, es decir, mujeres, jóvenes, población con bajos ingresos y residentes rurales. A pesar de que se ha logrado ampliar la tenencia de cuentas en instituciones financieras por parte de estos grupos más desfavorecidos, en muchos casos hay cuentas que permanecen totalmente inactivas. En concreto, según los datos más recientes extraídos de la Global Financial Inclusion database (Global Findex ${ }^{9}$ ), en 2014 un 62\% de la población mundial adulta estaba bancarizada, frente a un $51 \%$ en 2011. Dicho con otras palabras, en el transcurso de tres años, se ha reducido en un 20\% el número de personas que no tiene una cuenta en una institución financiera (incluye bancos, cooperativas, cooperativas, instituciones de microfinanzas, entre otros), ni accede a los servicios financieros formales a través del móvil.

Si se analiza la situación distribuyendo los países de acuerdo al Producto Nacional Bruto per cápita, la proporción de la población que está dentro del sistema

\footnotetext{
${ }^{9}$ Elaborada dentro del Grupo del Banco Mundial, se considera por el momento la base de datos sobre inclusión financiera más completa del mundo. Proporciona indicadores que cuantifican el modo en que las personas ahorran, se endeudan, efectúan pagos y gestionan los riesgos en más de 140 economías. Estos indicadores se calculan a partir de los datos obtenidos a través de encuestas realizadas a más de 150.000 adultos mayores de 15 años, seleccionados al azar y representativos de cada país. Hasta la fecha, se han realizado dos rondas de entrevistas, en 2011 y 2014, cuyos principales resultados están recogidos en dos informes del Banco Mundial: Demirgüç-Kunt y Klapper (2012) y Demirgüç-Kunt et al. (2015). Además, la base de datos completa está accesible de forma gratuita en: http://datatopics.worldbank.org/financialinclusion.
} 
financiero estructurado se sitúa alrededor del 91\% (un 85\% en 2011) en los países con alto nivel de renta, mientras que las cifras no llegan al 54\% (un 41\% en 2011) en las economías en desarrollo. También hay diferencias considerables por área geográfica, situándose en el extremo superior los países de la OCDE (94\%) y en el extremo inferior la zona de Oriente Medio (14\%). Si bien, es cierto que todas las regiones han mejorado su nivel de participación en el sistema financiero.

En la siguiente tabla se muestran varios indicadores de inclusión financiera, pertenecientes al mencionado Global Findex de 2014, agrupados por zona geográfica. Como puede apreciarse persisten las desigualdades en los niveles de exclusión financiera en el ámbito internacional, tanto en el acceso a los servicios financieros como en el uso que hacen de los mismos.

\section{Tabla 3. Indicadores de Inclusión Financiera - 2014 (\% de población mayor de 15 años)}

\begin{tabular}{|c|c|c|c|c|c|c|c|c|}
\hline (En porcentaje) & $\begin{array}{l}\frac{0}{0} \\
\frac{0}{0} \\
\frac{0}{00} \\
0 \\
0 \\
0 \\
0\end{array}$ & 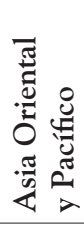 & 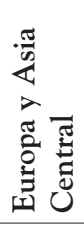 & ชิ & 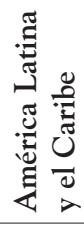 & 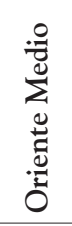 & 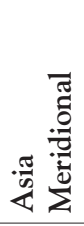 & 宽 \\
\hline Poseedores de una cuenta ${ }^{(1)}$ & 61,5 & 69,0 & 51,4 & 94,0 & 51,4 & 14,2 & 46,4 & 34,2 \\
\hline - Mujeres & 58,1 & 67,0 & 47,4 & 93,8 & 48,6 & 9,2 & 37,4 & 29,9 \\
\hline - Jóvenes (15-24 años) & 46,3 & 60,7 & 35,6 & 84,1 & 37,4 & 7,6 & 36,7 & 25,9 \\
\hline - Hogares más pobres & 54,0 & 60,9 & 44,2 & 90,6 & 41,2 & 7,3 & 38,1 & 24,6 \\
\hline - Residentes rurales & 56,7 & 64,5 & 45,7 & 93,8 & 46,0 & 10,7 & 43,5 & 29,2 \\
\hline Tienen tarjeta bancaria (débito) & 40,1 & 42,9 & 36,9 & 79,7 & 40,4 & 8,5 & 18,0 & 17,9 \\
\hline $\begin{array}{l}\text { Utilizan la cuenta para: } \\
\text { - recibir el salario }\end{array}$ & 17,7 & 15,1 & 22,5 & 44,3 & 18,0 & 3,3 & 3,5 & 7,3 \\
\hline - recibir transferencias del gobierno & 8,2 & 8,1 & 7,3 & 17,2 & 9,0 & 0,9 & 3,1 & 3,8 \\
\hline - pagar suministros (luz, agua,....) & 16,7 & 11,8 & 12,5 & 61,1 & 6,3 & 0,2 & 2,7 & 2,8 \\
\hline Uso de la tarjeta bancaria ${ }^{(2)}$ & & & & & & & & \\
\hline - Tarjeta de débito & 23,2 & 14,8 & 22,9 & 65,3 & 27,7 & 3,3 & 8,5 & 8,7 \\
\hline - Tarjeta de crédito & 15,1 & 10,8 & 14,9 & 46,7 & 18,0 & 1,5 & 2,6 & 1,9 \\
\hline Ahorros en entidad financiera ${ }^{(2)}$ & 27,4 & 36,5 & 8,4 & 51,6 & 13,5 & 4,0 & 12,7 & 15,9 \\
\hline Crédito de una entidad financiera ${ }^{(2)}$ & 10,7 & 11,0 & 12,4 & 18,4 & 11,3 & 5,6 & 6,4 & 6,3 \\
\hline
\end{tabular}

Fuente: elaboración propia con datos de World Bank (2015).

(1) Porcentaje de encuestados que dicen tener una cuenta (individual o compartida) en una entidad financiera o de servicios de dinero móvil en los últimos 12 meses.

(2) Con actividad en los últimos 12 meses. 
De igual forma, se detectan desigualdades en el comportamiento de los hogares en función de su nivel de renta. En 2014, solo el 54\% de los adultos pertenecientes a los hogares más pobres del planeta tenía una cuenta en una institución financiera, mientras que ese porcentaje ascendía a un $67 \%$ en los hogares más ricos ${ }^{10}$. No obstante, la evolución ha sido realmente positiva en estos últimos tres años. En los países en desarrollo, se ha conseguido que la proporción de hogares ricos con acceso a los servicios financieros formales haya aumentado en 11 puntos porcentuales desde 2011, y este incremento es todavía mayor en los hogares con nivel de renta más baja ( $17 \%)$.

Por otra parte, son evidentes las diferencias por género, edad, nivel de ingresos, lugar de residencia o nivel educativo ${ }^{11}$. El Banco Mundial se hace eco de estas desigualdades incluso en sus folletos divulgativos sobre inclusión financiera.

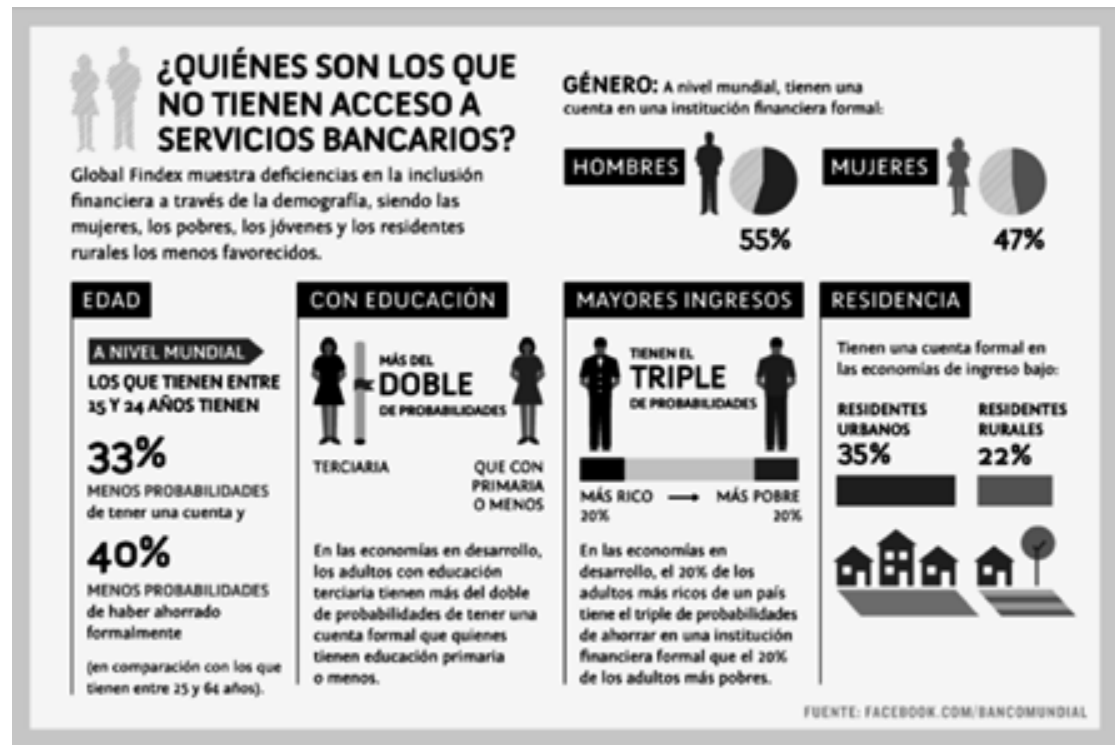

Si analizamos la variable género con más detalle, observamos que las diferencias entre hombres y mujeres se ponen de manifiesto en la tenencia de cuentas en entidades financieras y en los hábitos de uso de los servicios financieros. Las instituciones internacionales reconocen que el acceso de la mujer a los servicios financieros es particularmente importante, porque le proporcionaría un lugar dónde colocar sus ahorros y el control sobre las finanzas del hogar (World Bank, 2015).

${ }^{10}$ Según la clasificación realizada en la base de datos del Banco Mundial, los hogares están distribuidos en dos grupos: $40 \%$ más pobres y $60 \%$ más ricos. Para un mayor detalle sobre la metodología de recogida y tratamiento de la información, consúltese: http://datatopics.worldbank.org/financialinclusion.

${ }^{11}$ Véase a este respecto el trabajo realizado por Allen et al. (2012), dónde se analizan en profundidad estas variables. 
En el gráfico 1, se muestran los porcentajes de acceso a los servicios financieros formales de hombres y mujeres, agrupados por zona geográfica. Como puede verse en dicho gráfico la brecha de género permanece, aunque por hacer una lectura positiva de las cifras, las distancias se han reducido en 2014 respecto a los datos recogidos en 2011. Las desigualdades entre hombres y mujeres son más agudas en los países con menor nivel de renta o con mercados financieros menos desarrollados.

Por otra parte, si se examina el comportamiento de las mujeres que sí están bancarizadas, utilizan los productos de ahorro y de crédito con una frecuencia inferior a los hombres. Esto hace pensar que las diferencias no se deben sólo a las barreras de acceso, sino que responden a un comportamiento distinto como consumidor de servicios financieros.

En lo relativo al comportamiento particular de cada individuo, la base de datos Global Findex ofrece información sobre las diferencias conductuales de la población no bancarizada. En concreto, cuando se pregunta a los entrevistados sobre las razones que justifican que no tengan una cuenta en una entidad financiera, el tipo de respuesta es diverso. El principal motivo para no utilizar los servicios financieros en todas las zonas es la falta de dinero suficiente (30\% en 2014, 59\% en 2011), excepto en Europa y Asia Central. En segundo lugar, la respuesta más frecuente es que algún miembro de la familia tiene ya una cuenta ( $25 \%$ en 2014 , $30 \%$ en 2011). Adicionalmente, otras razones para no integrarse en el sistema financiero formal son: los altos costes de las cuentas, la distancia geográfica a las sucursales bancarias, la falta de documentación requerida, la falta de confianza o razones religiosas (World Bank, 2015; Demirgüç-Kunt y Klapper, 2013).

\section{Gráfico 1. Acceso a los servicios financieros formales (Año 2014 - diferencias de género)}

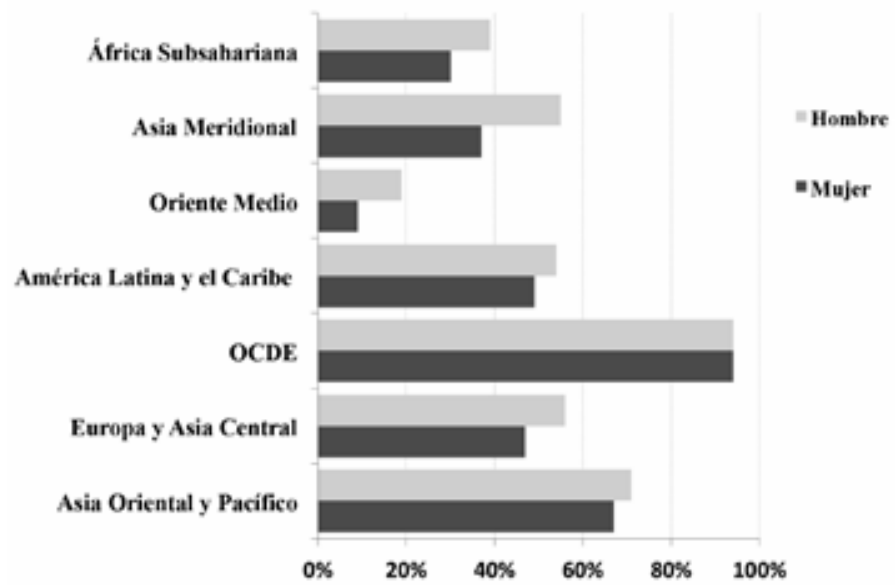

Fuente: elaboración propia basada en datos del World Bank (2015). 
Independientemente de la situación actual de la inclusión financiera, según el informe final del Global Findex 2014 (Demirgüç-Kunt et al., 2015), se han adoptado medidas que han resultado especialmente eficaces en la reducción de las denominadas barreras de exclusión financiera. Por citar algunas: la exigencia de que las entidades financieras ofrezcan cuentas básicas y de bajo coste, la reducción en la documentación requerida a los clientes o la implantación más extendida de las nuevas tecnologías. Sobre este último aspecto, sirva como ejemplo la experiencia de África. La región de África subsahariana es la única zona dónde más de un $10 \%$ de la población reconoce tener una cuenta de dinero móvil y, en el caso concreto de algunos países (Somalia, Tanzania, Uganda y Zimbabue, entre otros) es mayor la proporción de cuentas de dinero móvil que la tenencia de cuentas en instituciones financieras.

En cuanto a la tan comentada digitalización de los servicios financieros, se estima que si los pagos del Gobierno (transferencias y salarios) se realizasen en forma digital en lugar de en efectivo, se podría incrementar en 160 millones el número de personas con cuenta en entidades financieras. Por tanto, esta sería una vía adicional para fomentar la creación y uso de los servicios financieros formales, cuya responsabilidad recae en el sector público, pero en cuya tarea puede contribuir también el sector privado.

\section{REFLEXIONES FINALES}

La inclusión financiera se ha convertido en un elemento esencial en la Agenda 2030 para el Desarrollo Sostenible ${ }^{12}$, ya que se considera requisito indispensable para alcanzar un crecimiento económico sostenible, inclusivo y sostenido en el mundo. En concreto, desde numerosos organismos internacionales y nacionales, se ha reconocido que las políticas de inclusión financiera son imprescindibles para cumplir el objetivo de erradicación de la pobreza en todas sus formas, así como para reducir la desigualdad entre países y entre distintos segmentos de la población de cada país. En principio, parece ser que liberar el potencial económico y social de los más de 2.000 millones de personas más pobres del planeta, a través de un mayor y mejor acceso a los servicios financieros estructurados (formales), ayudaría a mejorar la estabilidad financiera global.

Todo ello justifica las iniciativas que se han puesto en marcha en los últimos años, tanto desde el sector público como privado, orientadas directamente a la reducción de la exclusión financiera en el ámbito internacional. Ahora bien, sería deseable que existiese una mejor coordinación entre las diferentes estrate-

\footnotetext{
2015.

${ }^{12}$ Recordemos que fue aprobada por la Asamblea General de Naciones Unidas en septiembre de
} 
gias y políticas de inclusión financiera, con vistas a conseguir actuaciones más efectivas y optimizar los esfuerzos realizados. Además, no hay que olvidar que las barreras para acceder a los servicios financieros formales en cada país y las necesidades de los individuos financieramente excluidos, no son las mismas para todos. Por tanto, no se pueden ofrecer soluciones homogéneas. Sirva como ejemplo, la iniciativa de la Alliance for Financial Inclusion plasmada en la denominada Declaración Maya, cuyo éxito radica en un marco de actuación flexible, en el cual se reconocen las particularidades de cada país miembro, así como las diferentes prioridades políticas. Y, esta flexibilidad ha hecho que los objetivos y planes de acción se establezcan a nivel nacional, en lugar de ser pautas estandarizadas para todos los países (AFI, 2013).

Conforme se ha comentado en los apartados anteriores, la evolución de la inclusión financiera está siendo muy positiva, aunque aún queda trabajo por hacer. Los principales grupos en exclusión financiera o "sin bancarizar" en el mundo siguen siendo: las mujeres, los jóvenes y los migrantes; alrededor de los cuales confluyen ciertas características comunes: bajos ingresos (o carencia de ellos), bajo nivel educativo general, falta de habilidades tecnológicas e insuficiente conocimiento de los productos financieros existentes.

Llegados a este punto, las autoridades públicas y los reguladores del sistema financiero internacional tienen por delante una serie de desafíos ${ }^{13}$, que podrían llegar a convertirse en oportunidades, como son:

- Inclusión financiera digital (innovación, supervisión y regulación). Es imprescindible desarrollar cauces para regular y supervisar los nuevos tipos de actores en el sector financiero, consecuencia de la implantación de las nuevas tecnologías y de los nuevos modelos de negocio (por ejemplo, las plataformas de crowdfunding y de peer-to-peer lending). Ya que, aunque proporcionan un mayor acceso a la financiación, no significa que automáticamente mejoren la estabilidad financiera sin deteriorar la inclusión financiera.

- Mejora de la protección al consumidor y de su educación financiera (conocimiento, habilidades y capacidad para decidir). Además de mejorar la educación financiera de la población, convendría estudiar más en profundidad la relación entre estabilidad financiera e inclusión financiera, ya que existen ciertas cautelas en los países en desarrollo sobre las consecuencias que los nuevos estándares globales para eliminar el riesgo sistémico en busca de la estabilidad financiera, puedan tener sobre la inclusión financiera.

\footnotetext{
${ }^{13}$ Estos retos están en línea de las prioridades fijadas en el reciente Plan de Acción para la inclusión financiera del G-20, a través del Global Partnership for Financial Inclusion (GPFI), cuyo informe completo está disponible en http://www.gpfi.org/publications/global-partnership-financial-inclusion-gpfi-china2016-priorities-paper.
} 
- Diversificación de las vías de financiación para las pequeñas y medianas empresas. No sólo se trata de llevar a cabo políticas inclusivas de los individuos, sino también de sus negocios.

- Reducir las desigualdades en el acceso a los servicios financieros, condicionadas por el género, la edad o el lugar de residencia.

- Fomentar el análisis y la recogida de datos integrales sobre inclusión financiera, desde el lado de la oferta y de la demanda, con vistas a elaborar indicadores comparables en el ámbito internacional.

\section{BIBLIOGRAFÍA}

AFI (2015), 2015 Maya Declaration Progress Report - Commitments into action. Alliance for Financial Inclusion, diciembre.

AFI (2013), Putting Financial Inclusion on the Global Map - The 2013 Maya Declaration Progress Report. Alliance for Financial Inclusion, septiembre.

AFI (2010), Financial inclusion measurement for regulators: Survey design and implementation. Alliance for Financial Inclusion, febrero.

ALLEN, F., DEMIRGÜÇ-KUNT, A., KLAPPER, L. y MARTÍNEZ PERÍA, M. S. (2012), "The Foundations of Financial Inclusion: Understanding Ownership and Use of Formal Accounts". Policy Research Working Paper, $\mathrm{n}^{\circ}$ 6290, World Bank, Washington.

ATKINSON, A. y MESSY, F. (2013), "Promoting Financial Inclusion through Financial Education: OECD/INFE Evidence, Policies and Practice". OECD Working Papers on Finance, Insurance and Private Pensions, $n^{\circ} 34$, OECD Publishing. CÁMARA, N. y TUESTA, D. (2015), "Factors that matter for financial inclusion: Evidence from Peru". AESTIMATIO The IEB International Journal of Finance, $n^{\circ} 10$, pp. 10-31.

DEMIRGÜÇ-KUNT, A., KLAPPER L., SINGER, D. y VAN OUDHEUSDEN, P. (2015), "The Global Findex Database 2014: Measuring Financial Inclusion around the World". Policy Research Working Paper, $\mathrm{n}^{\circ}$ 7255, World Bank, Washington.

DEMIRGÜÇ-KUNT, A. y KLAPPER, L. (2013), "Measuring Financial Inclusion: Explaining variation in use of financial services across and within countries". Brookings papers on Economic Activity, vol. 46 (issue 1- Spring), pp. 279-340.

DEMIRGÜÇ-KUNT, A. y KLAPPER, L. (2012), "Measuring Financial Inclusion: The Global Findex Database", Policy Research Working Paper, n 6025, World Bank, Washington. 
EESC (2013), Financial education for all: Financial education strategies and best practices within the European Union. European Economic and Social Committee, Brussels.

GHATAK, A. (2013), "Demand Side Factors Affecting Financial Inclusion". Research Journal of Social Science $\mathcal{E}$ Management, vol. 3 ( $\mathrm{n}^{\circ} 1$ ), mayo, pp. 176-185.

G-20 (2010), Innovative Financial Inclusion, Principles and Report on Innovative Financial Inclusion from the Access through Innovation Sub-Group of the G20 Financial Inclusion Experts Group, mayo.

GPFI (2011), Global Standard-Setting Bodies and Financial Inclusion for the Poor - Toward Proportionate Standards and Guidance, White Paper, octubre.

LUSARDI, A. y MITCHELL, O. S. (2013), "The Economic Importance of Financial Literacy: Theory and Evidence". NBER Working Paper $n^{\circ}$ 18952, abril.

OECD (2015), Toolkit for measuring Financial Literacy and Financial Inclusion. OECD/INFE, marzo. Disponible en http://www.oecd.org/finance/financial-education (última consulta: junio 2016).

OECD (2014), Progress Report on Financial Education. OECD/INFE, septiembre. Disponible en http://www.oecd.org/finance/financial-education/ OECD-INFE-FinEdG20-2014-ProgressReport.pdf (última consulta: junio 2016).

OECD (2013), Financial literacy and inclusion: Results of OECD/INFE survey across countries and by gender, junio. Disponible en https://www.oecd.org/ daf/fin/financial-education/TrustFund2013_OECD_INFE_Fin_Lit_and_ Incl_SurveyResults_by_Country_and_Gender.pdf (última consulta: junio 2016).

WORLD BANK (2015), The Little Data Book on Financial Inclusion 2015. World Bank, Washington. Disponible en http://dx.doi.org/10.1596/9781-4648-0552-3 (última consulta: abril 2016).

WORLD BANK (2014), Global Financial Development Report 2014: Financial Inclusion. World Bank, Washington. 